\title{
RENAL INVOLVEMENT IN GRAVES' DISEASE PATIENTS TREATED WITH BENZYLTHIOURACIL.
}

\author{
I.Oueslati, A.Melki, H.Kaaroud, I.Ben Nacef, N.Mchirgui, K.Khiari,N.Ben Abdallah. \\ Department of Endocrinology-Charles Nicolle Hospital, Tunis-Tunisia.
}

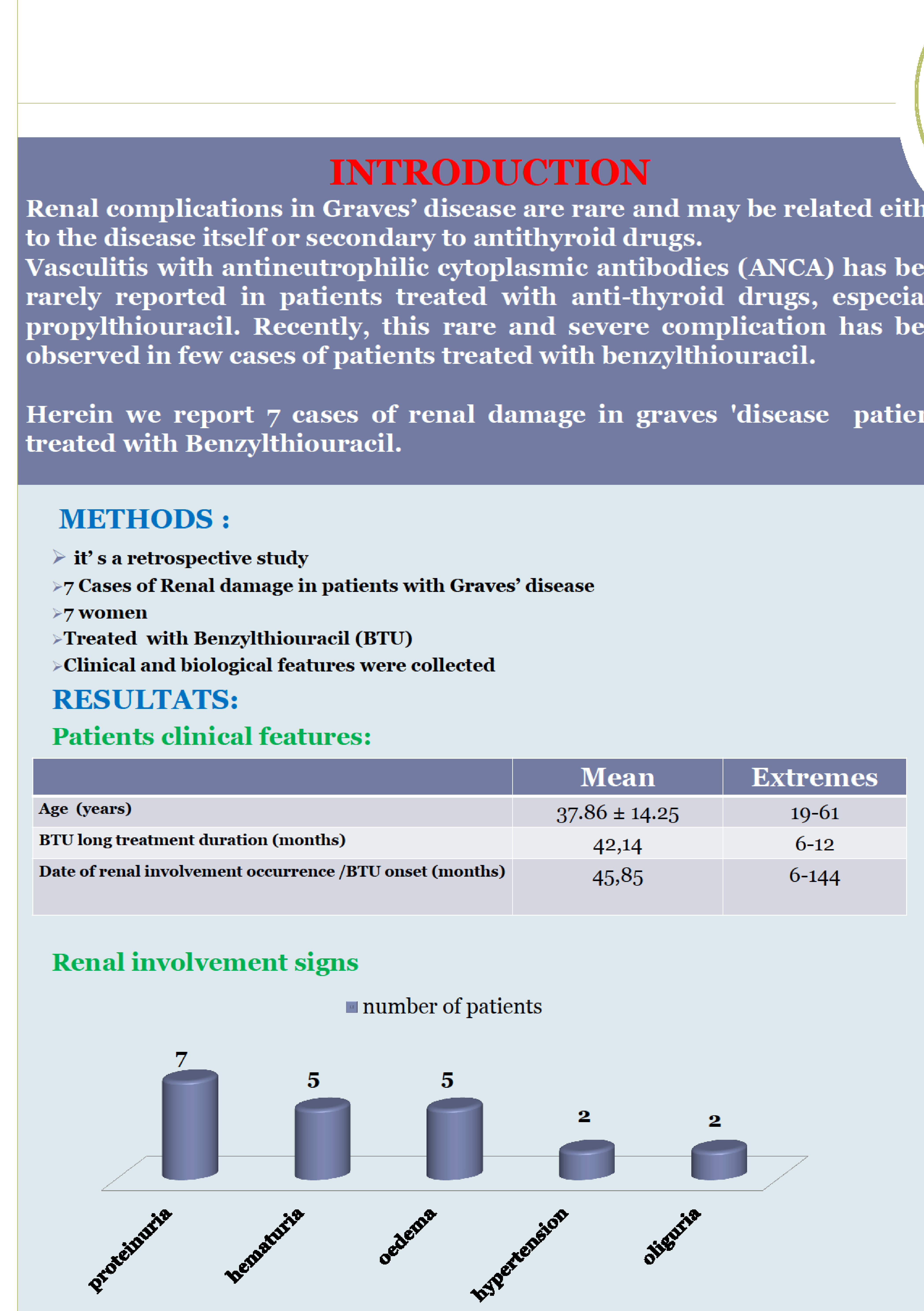

Extra-renal manifestations

number of patients

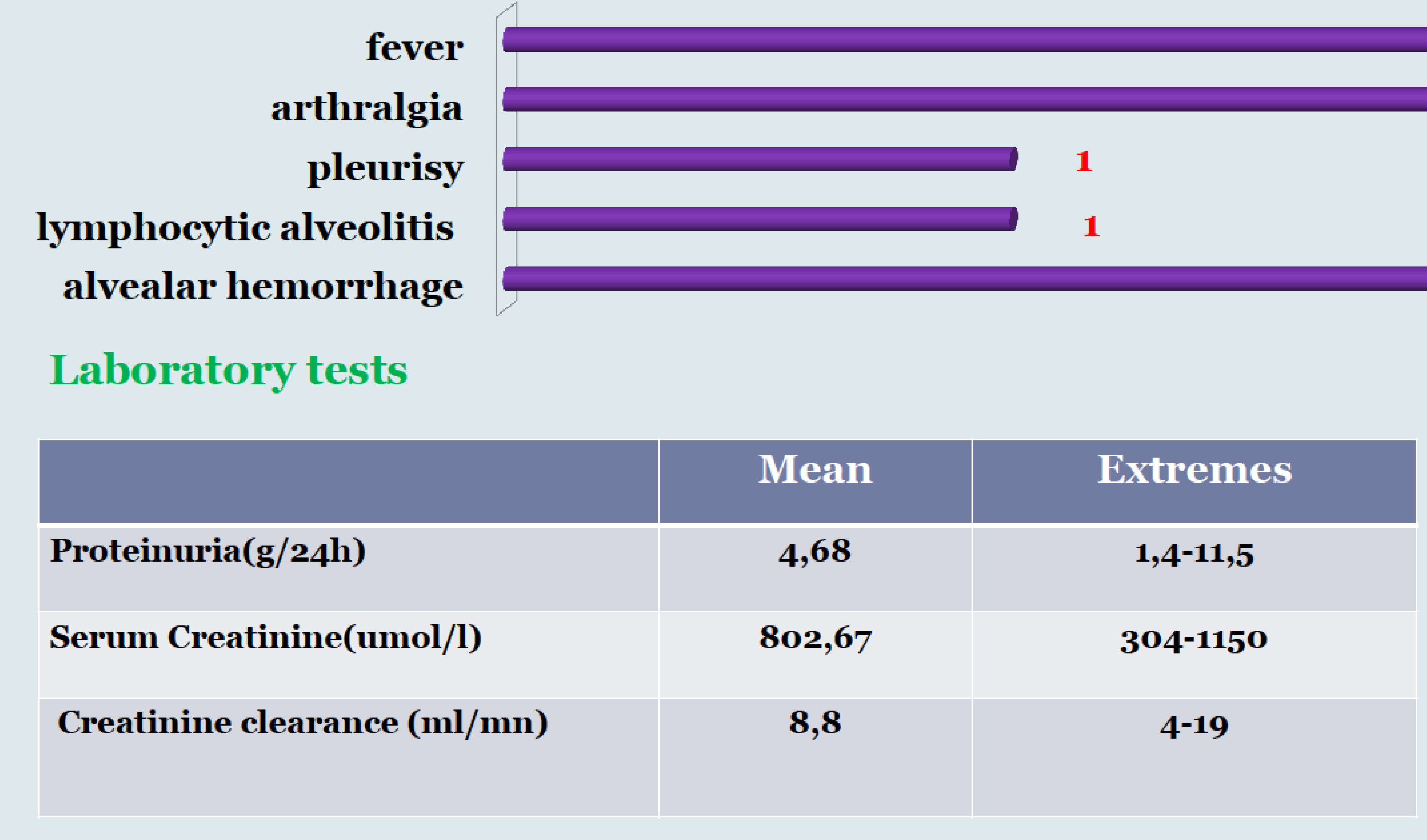

Thyroid status

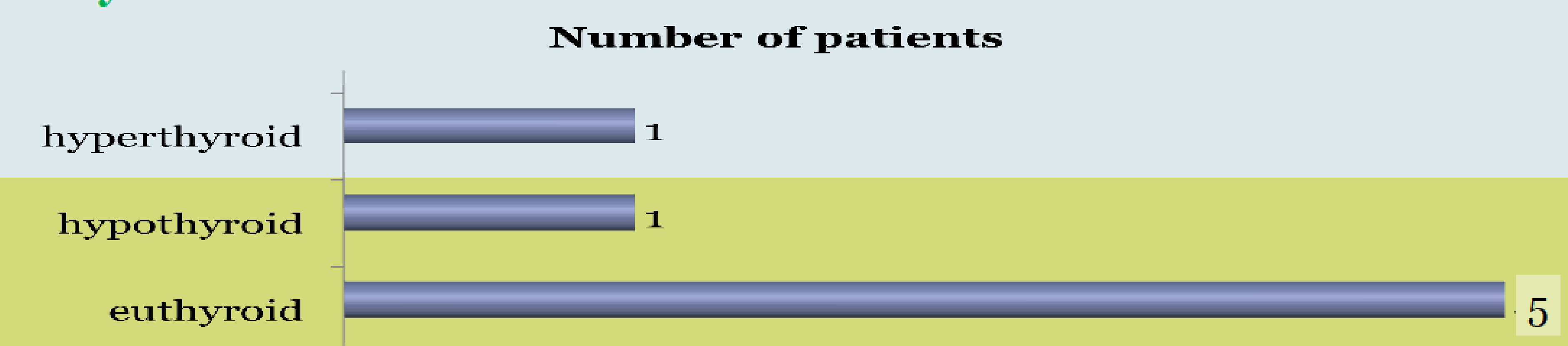

Anti-neutrophil cytoplasmic antibodies (ANCA) were positive in 6 patients and negative in one patient
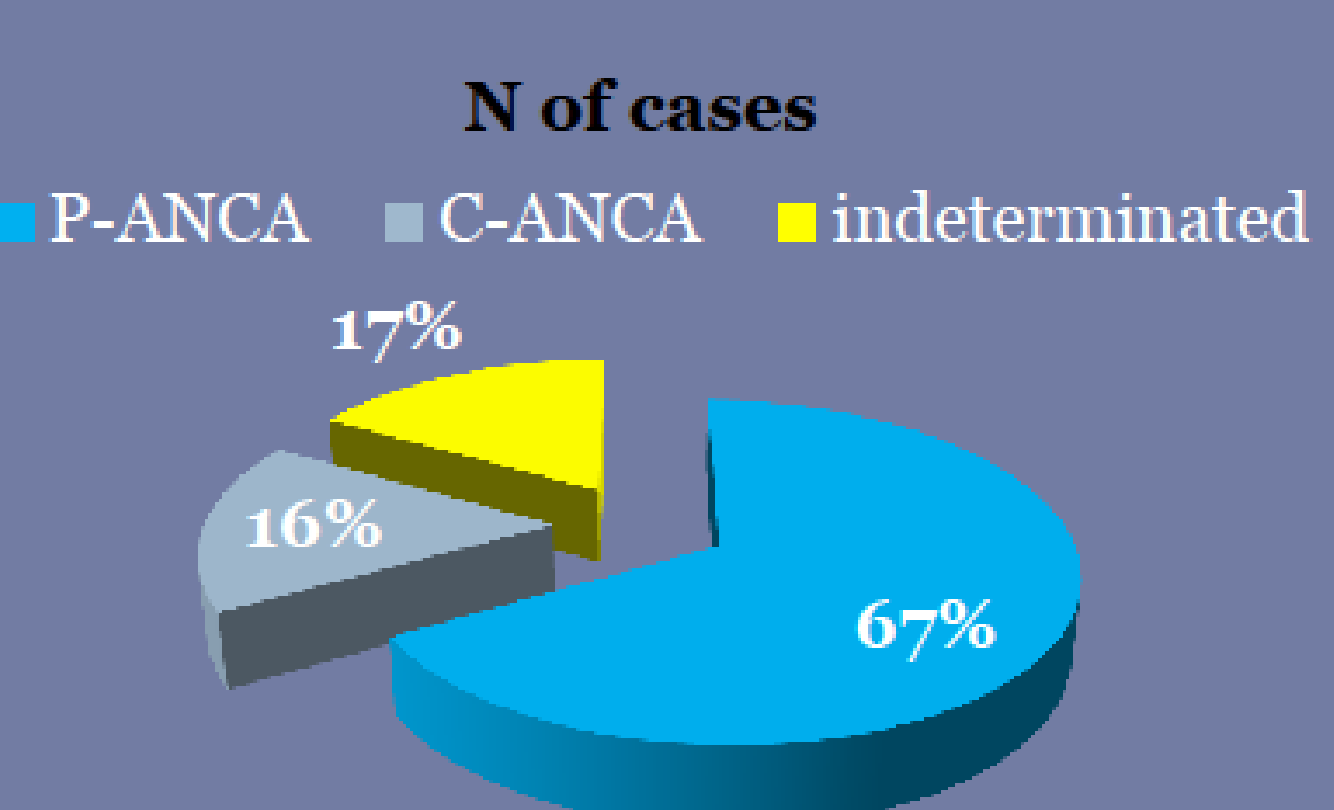